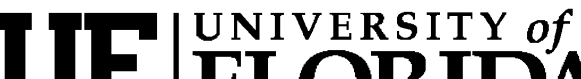 FLORIDA \\ IFAS Extension
}

\section{Dairy Business Analysis Project: 2005 Financial Summary 1}

\section{R. Giesy, L. Ely, \\ Introduction}

The Dairy Business Analysis Project (DBAP) was initiated in 1996 by the University of Florida in an effort to measure and document the financial performance of Florida dairy farms using standardized accounting measures. The University of Georgia has been a formal collaborator since 1998. A committee of dairy farmers appointed by Southeast Milk, Inc. oversees the project and helps direct its course. The DBAP website is http://dairy.ifas.ufl.edu/dbap.

Financial data for the year 2005 were collected from participating dairy farms and screened for completeness and validity. Each dairy farm then received a benchmark report detailing its financial results compared to the average results for the other participants and the six dairy farms with the highest net farm income per cwt. This benchmark report is discussed with the dairy farms to identify challenges and opportunities for improvement.

This publication is a summary of the financial performance of the dairy farms that participated in
2005. It is intended for general use by dairy farmers, the allied industry, and government and educational professionals.

\section{Data Collection and Accounting Methods}

Dairy farms in Florida and Georgia were asked to participate in DBAP. Participants were not a random sample of all dairy farms in the two states. The financial performance results in this publication are therefore not necessarily representative of the results of all dairy farms in Florida and Georgia.

Most of the data were collected by extension agents when visiting dairy farms using a standardized data collection spreadsheet. Occasionally, data were sent in by the dairy farms. The financial data were either entered into the spreadsheet on the farm or mailed in on paper copies of the spreadsheet.

The accounting methods followed the recommendations made by the Farm Financial Standards Council. ${ }^{3}$ All revenues and expenses were accrual adjusted. Cash receipts and expenses were therefore adjusted for changes in inventory, prepaid

1. This document is AN179, one of a series of the Department of Animal Sciences, Florida Cooperative Extension Service, Institute of Food and Agricultural Sciences, University of Florida. Published February 2007. Please visit the EDIS Web Site at http://edis.ifas.ufl.edu.

2. R. Giesy, Extension Agent IV; L. Ely, Professor, Department of Animal and Dairy Science, University of Georgia; B. Broaddus, Extension Agent I; C. Vann, Extension Agent II; A. Bell, Graduate Student; A. De Vries, Assistant Professor, Department of Animal Sciences; Cooperative Extension Service, Institute of Food and Agricultural Sciences, University of Florida, Gainesville, 32611 
expenses, accounts payable, and accounts receivable. Depreciation data were often taken from tax records. Asset valuation was based on market values if available, but the changes from January 1 to December 31 were kept small. Unpaid management was valued at $\$ 50,000$ per farm. Gain or loss on sale of purchased livestock resulted when livestock depreciation did not completely account for the gain or loss in the value of the purchased livestock during 2005. Appreciation resulted when machinery and building depreciation did not completely account for the gain or loss in the value of these capital assets during 2005. The bottom line of each dairy farm is its net farm income. Net farm income is the return to the owner and unpaid family members for their labor, management, and equity in the dairy farm. It is the total income available for owner's salary, new investments, taxes, and paying off principal.

All submitted data were carefully scrutinized and checked for completeness. The cash flow statement reconciles the net cash flow resulting from the reported operating, investing, and financing activities with the reported available cash on the January 1 and December 31 balance sheets. The equity statement reconciles the changes in equity through reported retained capital and valuation with the calculated equity on the balance sheets. The reconciliation attempts typically result in unresolved imbalances. Both cash flow imbalance and equity imbalance had to be less than $10 \%$ to be included in the summary results reported here.

All results in this publication are the simple averages of the statistics of the dairy farms with valid data. Every dairy farm has equal weight. For example, assume a 100-cow herd produced 1,700,000 lbs of milk (17,000 lbs/cow) and a 1000-cow herd produced 19,000,000 lbs of milk (19,000 lbs / cow $)$. Average milk yield per farm is $(19,000,000+$ $1,700,000) / 2=10,350,000 \mathrm{lbs}$ and average herd size is $(100+1000) / 2=550$ cows. The weighted average milk yield per cow is 10,350,000 $/ 550=$ $18,818 \mathrm{lbs} / \mathrm{cow}$. The simple average milk yield per cow is $(17,000+19,000) / 2=18,000 \mathrm{lbs} /$ cow .

Some definitions and calculation rules are as follows:
- Asset turnover ratio = total revenues $/$ average assets

- Assets = value of assets on the balance sheet

- Average assets = average of value of assets on January 1 and December 31

- Average equity = average of value of equity on January 1 and December 31

- Capital replacement and term debt repayment margin $=$ Net Farm Income from Operations $(\mathrm{NFIFO})+$ depreciation + interest on term debt - net social security and income taxes owner withdrawals - annual scheduled payments on term debt and capital leases

- Cash flow coverage ratio $=($ cash revenues cash expenses) / current liabilities

- Current assets $=$ short-term assets that can be utilized within one year

- Current liabilities = liabilities due within one year

- Current ratio = current assets $/$ current liabilities

- Debt to asset ratio = liabilities $/$ assets

- Debt to equity ratio = liabilities $/$ equity

- Depreciation expense ratio $=$ depreciation $/$ total revenue

- Equity = assets - liabilities

- Equity to asset ratio = equity $/$ assets

- FTE = full time equivalent worker, on average 54 hours per week

- Interest expense ratio $=$ interest paid $/$ total revenue

- Liabilities = value of liabilities on the balance sheet

- Net farm income = NFIFO + gain on sale of capital assets 
- NFIFO = net farm income from operations

- $\mathrm{NFIFO}$ ratio $=\mathrm{NFIFO} /$ total revenue

- Operating expense ratio $=($ total operating expenses - depreciation) / total revenue

- Operating profit margin ratio $=(\mathrm{NFIFO}+$ interest paid - unpaid management) / total revenues

- Rate of return on dairy assets $=(\mathrm{NFIFO}+$ interest paid - unpaid management) / average assets

- Rate of return on equity $=(\mathrm{NFIFO}-$ unpaid management) / average equity

- Std = standard deviation

- Term debt and capital lease coverage ratio $=$ (NFIFO + non dairy income + depreciation + interest paid on term debt - net social security and income taxes - owner withdrawals) / (annual scheduled payments on term debt and capital leases)

- Working capital $=$ current assets - current liabilities

\section{Results}

Summary results are presented in Tables 1.1 to 4.4 and Figures 1 to 5. Revenues and expenses may not add up due to rounding. In brief, twenty-one dairy farms were included in the summary results. Of these, 15 were located in Florida and 6 in Georgia. The average herd size of the participating dairies was 1045 cows and 538 heifers with $18,322 \mathrm{lbs}$. milk sold per cow. The average culling rate was $36 \%$. The average milk price was $\$ 18.24$. Average total revenues were $\$ 20.73$ per cwt. milk sold. Total expenses averaged $\$ 20.20$ per cwt. sold. The largest items were purchased feed, $\$ 7.22$, and personnel costs, $\$ 3.50$. Net farm income from operations averaged $\$ 0.53$ per cwt. sold. Net farm income per cwt. was $\$ 0.07$.

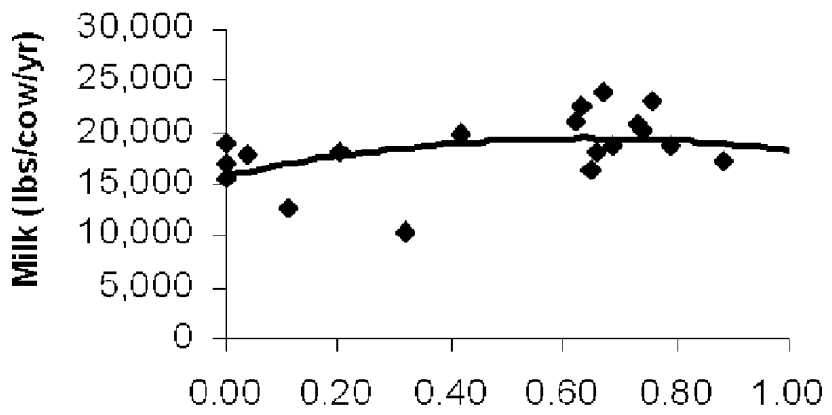

He ifers per cow

Figure 1. DBAP 2005 Summary - Milk production (lbs/cow/year) by heifers per cow.

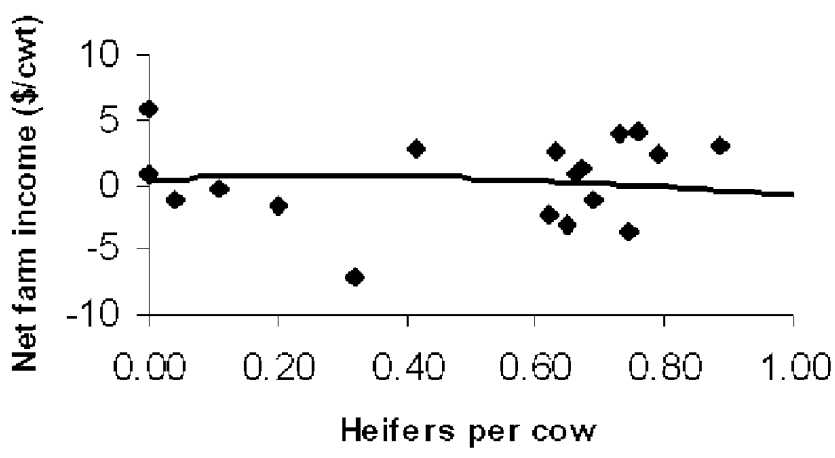

Figure 2. DBAP 2005 Summary - Net farm income (\$/cwt) by heifers per cow.

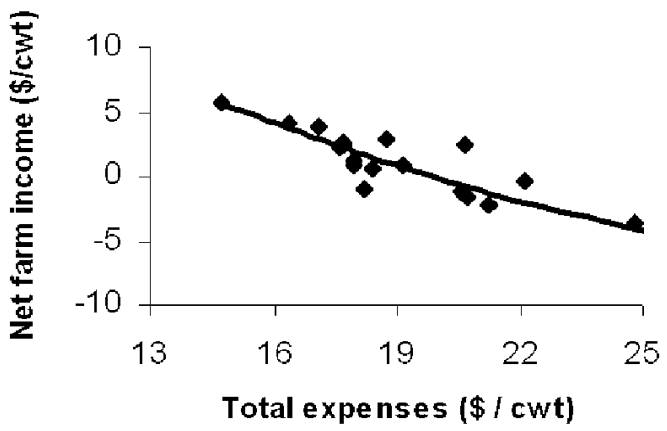

Figure 3. DBAP 2005 Summary - Net farm income (\$/cwt) by total expenses (\$/cwt).

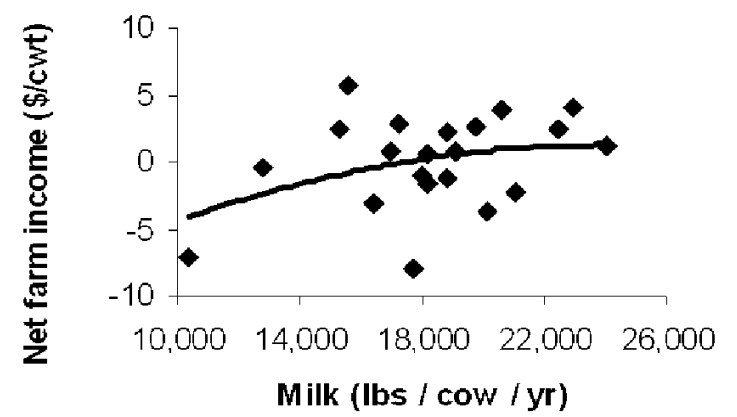

Figure 4. DBAP 2005 Summary - Net farm income (\$/cwt) by milk yield (lbs/cow/year). 


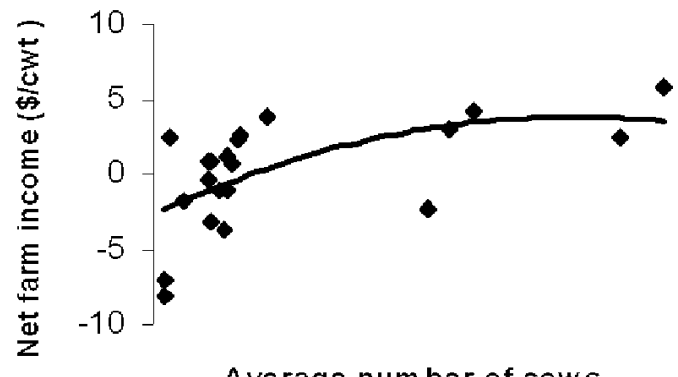

Average number of cows

Figure 5. DBAP 2005 Summary - Net farm income per cwt (\$) by average number of cows. The $\mathrm{x}$-axis is not displayed to avoid possible identification of dairy farms.

Table 1.1. DBAP 2005 Summary - Business size and production efficiency by state and overall average, median, and standard deviation.

\begin{tabular}{|c|c|c|c|c|c|}
\hline \multirow[b]{2}{*}{ Category } & \multicolumn{3}{|c|}{ Overall } & \multicolumn{2}{|c|}{ State Averages } \\
\hline & Average & Median & $\mathrm{Std}^{1}$ & Florida & Georgia \\
\hline Number of farms & 21 & 21 & 21 & 15 & 6 \\
\hline \multicolumn{6}{|l|}{ Business size: } \\
\hline - Average number of cows & 1,045 & 575 & 1,157 & 1,155 & 770 \\
\hline - Average number of heifers & 538 & 290 & 720 & 543 & 527 \\
\hline • Milk sold (million lbs) & 20.21 & 10.99 & 22.96 & 21.53 & 16.94 \\
\hline - $\mathrm{FTE}^{2}$ workers & 19 & 12 & 17 & 20 & 16 \\
\hline - Acres of pasture + cultivated land & 569 & 320 & 732 & 633 & 410 \\
\hline \multicolumn{6}{|l|}{ Production efficiency: } \\
\hline - Milk sold (lbs / cow / year) & 18,322 & 18,168 & 3,237 & 17,659 & 19,979 \\
\hline - Cows / FTE worker & 51 & 52 & 25 & 55 & 40 \\
\hline • Milk sold / FTE worker (million lbs) & 0.93 & 0.94 & 0.44 & 0.97 & 0.82 \\
\hline - Cull rate & $36 \%$ & $32 \%$ & $21 \%$ & $31 \%$ & $47 \%$ \\
\hline
\end{tabular}

${ }^{1}$ Standard deviation.

${ }^{2}$ Full-time equivalent. 
Table 1.2. DBAP 2005 Summary - Revenues and expenses by state and overall average, median, and standard deviation $(\$ / c w t)$.

\begin{tabular}{|c|c|c|c|c|c|}
\hline \multirow[b]{2}{*}{ Category } & \multicolumn{3}{|c|}{ Overall } & \multicolumn{2}{|c|}{ State Averages } \\
\hline & Average & Median & $S+d^{1}$ & Florida & Georgia \\
\hline Number of farms & 21 & 21 & 21 & 15 & 6 \\
\hline \multicolumn{6}{|l|}{ Revenues: } \\
\hline Milk sold & 18.24 & 18.28 & 0.62 & 18.38 & 17.89 \\
\hline Raised, leased cow sales & 0.89 & 0.33 & 1.41 & 0.60 & 1.62 \\
\hline Heifer sales & 0.45 & 0.36 & 0.44 & 0.50 & 0.33 \\
\hline Gain on purchased livestock sales & $(0.13)$ & $(0.06)$ & 0.83 & $(0.37)$ & 0.48 \\
\hline Other revenues & 1.28 & 0.78 & 1.45 & 1.30 & 1.23 \\
\hline Total revenues & 20.73 & 20.24 & 2.21 & 20.41 & 21.55 \\
\hline \multicolumn{6}{|l|}{ Expenses: } \\
\hline - Personnel & 3.50 & 3.08 & 1.42 & 3.43 & 3.69 \\
\hline - Purchased feed & 7.22 & 6.81 & 2.33 & 7.96 & 5.36 \\
\hline - Crops & 0.41 & 0.13 & 0.59 & 0.34 & 0.58 \\
\hline - Machinery & 1.11 & 1.00 & 0.75 & 1.18 & 0.94 \\
\hline - Livestock & 2.01 & 1.92 & 0.90 & 1.99 & 2.06 \\
\hline - Milk marketing & 1.22 & 1.30 & 0.24 & 1.19 & 1.32 \\
\hline - Buildings and land & 0.74 & 0.44 & 1.01 & 0.52 & 1.30 \\
\hline - Interest & 0.67 & 0.56 & 0.61 & 0.69 & 0.61 \\
\hline \multicolumn{6}{|l|}{ Depreciation: } \\
\hline - Livestock & 1.11 & 0.97 & 1.07 & 1.11 & 1.12 \\
\hline - Machinery & 0.81 & 0.40 & 0.85 & 0.76 & 0.95 \\
\hline - Buildings & 0.39 & 0.25 & 0.42 & 0.37 & 0.44 \\
\hline Other expenses & 1.01 & 1.03 & 0.38 & 1.06 & 0.89 \\
\hline Total expenses & 20.20 & 18.73 & 3.80 & 20.59 & 19.25 \\
\hline Net farm income from operations & 0.53 & 0.84 & 3.20 & $(0.18)$ & 2.30 \\
\hline Gain on sale of capital assets & $(0.46)$ & 0.00 & 1.37 & $(0.03)$ & $(1.56)$ \\
\hline Net farm income & 0.07 & 0.84 & 3.54 & $(0.20)$ & 0.74 \\
\hline
\end{tabular}

\footnotetext{
${ }^{1}$ Standard deviation
} 
Table 1.3. DBAP 2005 Summary - Financial performance by state and overall average, median, and standard deviation.

\begin{tabular}{|c|c|c|c|c|c|}
\hline \multirow[b]{2}{*}{ Category } & \multicolumn{3}{|c|}{ Overall } & \multicolumn{2}{|c|}{ State Averages } \\
\hline & Average & Median & $S+d^{1}$ & Florida & Georgia \\
\hline Number of farms & 21 & 21 & 21 & 15 & 6 \\
\hline \multicolumn{6}{|l|}{ Liquidity: } \\
\hline - Current ratio & 5.78 & 0.61 & 17.47 & 1.53 & 16.42 \\
\hline - Working capital (\$) & 123,069 & 21,375 & 673,678 & $(23,839)$ & 490,338 \\
\hline \multicolumn{6}{|l|}{ Solvency: } \\
\hline - Debt to asset ratio & 0.39 & 0.34 & 0.26 & 0.40 & 0.38 \\
\hline - Equity to asset ratio & 0.61 & 0.66 & 0.26 & 0.60 & 0.62 \\
\hline $\begin{array}{l}\text { - Debt to equity ratio }{ }^{2} \\
\text { Profitability: }\end{array}$ & 2.47 & 0.52 & 5.88 & 1.75 & 4.26 \\
\hline - Rate of return on assets & 0.04 & 0.04 & 0.10 & 0.03 & 0.09 \\
\hline - Rate of return on equity & 0.02 & 0.02 & 0.35 & $(0.06)$ & 0.24 \\
\hline - Operating profit margin ratio & 0.02 & 0.03 & 0.17 & $(0.01)$ & 0.08 \\
\hline \multicolumn{6}{|l|}{ Financial efficiency: } \\
\hline - Asset turnover rate & 0.78 & 0.81 & 0.37 & 0.80 & 0.71 \\
\hline - Operating expense ratio & 0.83 & 0.84 & 0.15 & 0.86 & 0.75 \\
\hline - Depreciation expense ratio & 0.11 & 0.10 & 0.06 & 0.11 & 0.11 \\
\hline - Interest expense ratio & 0.03 & 0.03 & 0.03 & 0.03 & 0.03 \\
\hline - NFIFO ratio ${ }^{3}$ & 0.03 & 0.04 & 0.15 & $(0.01)$ & 0.10 \\
\hline \multicolumn{6}{|l|}{ Repayment capacity: } \\
\hline - Cash flow coverage ratio & 5.96 & 0.58 & 19.28 & 0.71 & 19.08 \\
\hline - Term debt coverage ratio ${ }^{4}$ & 2.90 & 0.42 & 7.30 & 3.77 & 0.72 \\
\hline - Capital replacement margin ${ }^{5}(\$)$ & 321,516 & 69,895 & 971,525 & 193,709 & 641,032 \\
\hline
\end{tabular}

${ }^{1}$ Standard deviation.

${ }^{2}$ One dairy farm had negative equity.

${ }^{3}$ Net farm income from operations ratio.

${ }^{4}$ Term debt and capital lease coverage ratio.

${ }^{5}$ Capital replacement and term debt repayment margin. 
Table 1.4. DBAP 2005 Summary - Balance sheet by state and overall average, median, and standard deviation (\$/cow).

\begin{tabular}{|c|c|c|c|c|c|}
\hline \multirow[b]{2}{*}{ Category } & \multicolumn{3}{|c|}{ Overall } & \multicolumn{2}{|c|}{ State Averages } \\
\hline & Average & Median & $\mathrm{Std}^{1}$ & Florida & Georgia \\
\hline Number of farms & 21 & 21 & 21 & 15 & 6 \\
\hline \multicolumn{6}{|c|}{ Balance sheet (January 1): } \\
\hline - Current assets & 428 & 306 & 268 & 316 & 709 \\
\hline - Total assets & 6,800 & 4,176 & 5,763 & 5,502 & 10,044 \\
\hline - Current liabilities & 507 & 408 & 379 & 581 & 324 \\
\hline - Total liabilities & 1,810 & 1,825 & 1,163 & 1,668 & 2,164 \\
\hline - Equity & 4,990 & 2,415 & 6,108 & 3,834 & 7,880 \\
\hline \multicolumn{6}{|c|}{ Balance sheet (December 31): } \\
\hline - Current assets & 487 & 368 & 317 & 425 & 641 \\
\hline - Total assets & 6,668 & 4,733 & 5,315 & 5,365 & 9,925 \\
\hline - Current liabilities & 386 & 344 & 414 & 521 & 49 \\
\hline - Total liabilities & 1,896 & 1,914 & 1,159 & 1,854 & 2,001 \\
\hline • Equity & 4,772 & 2,657 & 5,724 & 3,511 & 7,924 \\
\hline
\end{tabular}

\footnotetext{
${ }^{1}$ Standard deviation
} 
Table 2.1. DBAP 2005 Summary - Business size and production efficiency by average number of cows and milk per cow.

\begin{tabular}{|c|c|c|c|c|c|c|}
\hline \multirow[b]{3}{*}{ Category } & \multicolumn{3}{|c|}{ Average number of cows } & \multicolumn{3}{|c|}{ Milk yield (Ibs/cow/year) } \\
\hline & \multicolumn{3}{|c|}{$446-$} & \multicolumn{3}{|c|}{$17,300-$} \\
\hline & $<446$ & 670 & $>670$ & $<17,300$ & 19,500 & $>19,500$ \\
\hline Number of farms & 7 & 7 & 7 & 7 & 7 & 7 \\
\hline \multicolumn{7}{|l|}{ Business size: } \\
\hline - Average number of cows & 261 & 562 & 2,312 & 1,117 & 447 & 1,571 \\
\hline - Average number of heifers & 50 & 348 & 1,218 & 363 & 215 & 1,038 \\
\hline • Milk sold (million lbs) & 4.24 & 10.86 & 45.54 & 17.84 & 8.29 & 34.51 \\
\hline - FTE workers & 7 & 14 & 36 & 15 & 9 & 33 \\
\hline - Acres of pasture + cultivated land & 142 & 397 & 1,169 & 613 & 298 & 798 \\
\hline \multicolumn{7}{|l|}{ Production efficiency: } \\
\hline - Milk sold (lbs/cow/year) & 15,777 & 19,225 & 19,963 & 14,950 & 18,420 & 21,594 \\
\hline - Cows / FTE worker & 37 & 49 & 66 & 58 & 47 & 47 \\
\hline - Milk sold / FTE worker (million lbs) & 0.59 & 0.93 & 1.26 & 0.89 & 0.88 & 1.02 \\
\hline - Cull rate & $42 \%$ & $34 \%$ & $31 \%$ & $44 \%$ & $34 \%$ & $29 \%$ \\
\hline
\end{tabular}

Table 2.2. DBAP 2005 Summary - Revenues and expenses by average number of cows and milk per cow (\$/cwt).

\begin{tabular}{|c|c|c|c|c|c|c|}
\hline \multirow[b]{3}{*}{ Category } & \multicolumn{3}{|c|}{ Average number of cows } & \multicolumn{3}{|c|}{ Milk yield (lbs/cow/year) } \\
\hline & \multicolumn{3}{|c|}{$446-$} & \multicolumn{3}{|c|}{$17,300-$} \\
\hline & $<446$ & 670 & $>670$ & $<17,300$ & 19,500 & $>19,500$ \\
\hline Number of farms & 7 & 7 & 7 & 7 & 7 & 7 \\
\hline \multicolumn{7}{|l|}{ Revenues: } \\
\hline - Milk sold & 17.95 & 18.14 & 18.63 & 18.32 & 18.08 & 18.32 \\
\hline - Raised, leased cow sales & 1.08 & 0.96 & 0.63 & 1.47 & 0.37 & 0.84 \\
\hline - Heifer sales & 0.38 & 0.34 & 0.64 & 0.37 & 0.36 & 0.63 \\
\hline - Gain on purchased livestock sales & 0.14 & $(0.46)$ & $(0.07)$ & 0.07 & $(0.43)$ & $(0.03)$ \\
\hline - Other revenues & 2.01 & 1.22 & 0.61 & 2.23 & 1.08 & 0.52 \\
\hline - Total revenues & 21.57 & 20.20 & 20.44 & 22.46 & 19.46 & 20.29 \\
\hline \multicolumn{7}{|l|}{ Expenses: } \\
\hline - Personnel & 4.04 & 3.63 & 2.85 & 3.31 & 3.96 & 3.24 \\
\hline - Purchased feed & 7.31 & 7.36 & 6.99 & 7.92 & 7.28 & 6.46 \\
\hline - Crops & 0.29 & 0.72 & 0.22 & 0.37 & 0.32 & 0.54 \\
\hline - Machinery & 0.95 & 1.53 & 0.85 & 0.93 & 1.04 & 1.35 \\
\hline - Livestock & 2.16 & 1.92 & 1.96 & 2.23 & 1.91 & 1.89 \\
\hline - Milk marketing & 1.34 & 1.06 & 1.27 & 1.29 & 1.17 & 1.22 \\
\hline - Buildings and land & 1.37 & 0.57 & 0.29 & 1.00 & 0.96 & 0.27 \\
\hline - Interest & 0.93 & 0.52 & 0.55 & 0.93 & 0.45 & 0.61 \\
\hline \multicolumn{7}{|l|}{ Depreciation: } \\
\hline - Livestock & 1.67 & 0.67 & 0.99 & 1.70 & 0.92 & 0.71 \\
\hline - Machinery & 0.88 & 1.14 & 0.42 & 0.63 & 0.75 & 1.05 \\
\hline - Buildings & 0.23 & 0.53 & 0.41 & 0.41 & 0.09 & 0.66 \\
\hline - Other expenses & 1.05 & 1.12 & 0.86 & 1.05 & 1.00 & 0.98 \\
\hline - Total expenses & 22.22 & 20.75 & 17.65 & 21.78 & 19.86 & 18.98 \\
\hline - Net farm income from operations & $(0.65)$ & $(0.55)$ & 2.79 & 0.68 & $(0.40)$ & 1.31 \\
\hline - Gain on sale of capital assets & $(1.23)$ & $(0.17)$ & 0.00 & $(0.50)$ & $(0.77)$ & $(0.12)$ \\
\hline - Net farm income & $(1.88)$ & $(0.72)$ & 2.79 & 0.18 & $(1.17)$ & 1.19 \\
\hline
\end{tabular}


Table 2.3. DBAP 2005 Summary - Financial performance by average number of cows and milk per cow.

\begin{tabular}{|c|c|c|c|c|c|c|}
\hline \multirow[b]{2}{*}{ Category } & \multicolumn{3}{|c|}{ Average number of cows } & \multicolumn{3}{|c|}{ Milk yield (lbs/cow/year) } \\
\hline & $<446$ & $446-670$ & $>670$ & $<17,300$ & $\begin{array}{l}17,300- \\
19,500\end{array}$ & $>19,500$ \\
\hline Number of farms & 7 & 7 & 7 & 7 & 7 & 7 \\
\hline \multicolumn{7}{|l|}{ Liquidity: } \\
\hline - Current ratio & 0.46 & 12.66 & 4.22 & 1.77 & 0.41 & 15.16 \\
\hline - Working capital $(\$)$ & 24,649 & 7,679 & 336,879 & 207,250 & $(32,694)$ & 194,650 \\
\hline \multicolumn{7}{|l|}{ Solvency: } \\
\hline - Debt to asset ratio & 0.48 & 0.30 & 0.40 & 0.38 & 0.43 & 0.36 \\
\hline - Equity to asset ratio & 0.52 & 0.70 & 0.60 & 0.62 & 0.57 & 0.64 \\
\hline - Debt to equity ratio ${ }^{1}$ & 5.99 & 0.65 & 0.77 & 0.79 & 5.92 & 0.70 \\
\hline \multicolumn{7}{|l|}{ Profitability: } \\
\hline - Rate of return on assets & $(0.01)$ & $(0.01)$ & 0.15 & 0.04 & $(0.00)$ & 0.09 \\
\hline - Rate of return on equity & $(0.08)$ & $(0.05)$ & 0.20 & 0.02 & $(0.07)$ & 0.12 \\
\hline - Operating profit margin ratio & $(0.08)$ & $(0.02)$ & 0.15 & 0.01 & $(0.04)$ & 0.08 \\
\hline \multicolumn{7}{|l|}{ Financial efficiency: } \\
\hline - Asset turnover rate & 0.74 & 0.67 & 0.93 & 0.77 & 0.83 & 0.73 \\
\hline - Operating expense ratio & 0.86 & 0.89 & 0.75 & 0.81 & 0.90 & 0.79 \\
\hline - Depreciation expense ratio & 0.13 & 0.11 & 0.09 & 0.12 & 0.09 & 0.12 \\
\hline - Interest expense ratio & 0.04 & 0.03 & 0.03 & 0.04 & 0.02 & 0.03 \\
\hline - NFIFO ratio ${ }^{2}$ & $(0.03)$ & $(0.02)$ & 0.13 & 0.03 & $(0.02)$ & 0.06 \\
\hline \multicolumn{7}{|l|}{ Repayment capacity: } \\
\hline - Cash flow coverage ratio & 0.15 & 13.03 & 4.68 & 0.57 & 0.74 & 16.56 \\
\hline - Term debt coverage ratio $^{3}$ & 0.53 & 1.49 & 6.67 & 5.11 & 1.30 & 2.28 \\
\hline - Capital replacement margin ${ }^{4}(\$)$ & $(1,170)$ & 69,793 & 895,924 & 342,885 & 35,085 & 586,576 \\
\hline
\end{tabular}

${ }^{1}$ One dairy farm had negative equity.

${ }^{2}$ Net farm income from operations ratio.

${ }^{3}$ Term debt and capital lease coverage ratio.

${ }^{4}$ Capital replacement and term debt repayment margin. 
Table 2.4. DBAP 2005 Summary - Balance sheet by average number of cows and milk per cow $(\$ / \mathrm{cow})$.

\begin{tabular}{|c|c|c|c|c|c|c|}
\hline \multirow[b]{2}{*}{ Category } & \multicolumn{3}{|c|}{ Average number of cows } & \multicolumn{3}{|c|}{ Milk yield (lbs/cow/year) } \\
\hline & $<446$ & $\begin{array}{c}446- \\
670\end{array}$ & $>670$ & $<17,300$ & $\begin{array}{c}17,300- \\
19,500\end{array}$ & $>19,500$ \\
\hline Number of farms & 7 & 7 & 7 & 7 & 7 & 7 \\
\hline \multicolumn{7}{|c|}{ Balance sheet (January 1): } \\
\hline - Current assets & 336 & 350 & 599 & 334 & 291 & 660 \\
\hline - Total assets & 8,343 & 7,651 & 4,405 & 5,512 & 6,846 & 8,042 \\
\hline - Current liabilities & 471 & 453 & 599 & 491 & 547 & 484 \\
\hline - Total liabilities & 1,877 & 1,604 & 1,949 & 1,858 & 1,374 & 2,197 \\
\hline - Equity & 6,466 & 6,047 & 2,457 & 3,654 & 5,471 & 5,844 \\
\hline \multicolumn{7}{|c|}{ Balance sheet (December 31): } \\
\hline - Current assets & 374 & 311 & 775 & 541 & 242 & 676 \\
\hline - Total assets & 8,526 & 6,726 & 4,751 & 5,715 & 7,017 & 7,271 \\
\hline - Current liabilities & 223 & 304 & 631 & 410 & 259 & 490 \\
\hline - Total liabilities & 2,061 & 1,623 & 2,004 & 2,015 & 1,504 & 2,169 \\
\hline - Equity & 6,465 & 5,102 & 2,747 & 3,700 & 5,513 & 5,102 \\
\hline
\end{tabular}


Table 3.1. DBAP 2005 Summary - Business size and production efficiency by net farm income per cwt and rate of return on assets.

\begin{tabular}{|c|c|c|c|c|c|c|}
\hline \multirow[b]{3}{*}{ Category } & \multicolumn{3}{|c|}{ Net farm income (\$/cwt) } & \multicolumn{3}{|c|}{ Rate of return on assets (\%) } \\
\hline & \multicolumn{3}{|c|}{$\$(1.13)$} & \multicolumn{3}{|c|}{ (3)\% - } \\
\hline & $<\$(1.13)$ & $-\$ 2.30$ & $>\$ 2.30$ & $(3) \%$ & $9.5 \%$ & $>9.5 \%$ \\
\hline Number of farms & 7 & 7 & 7 & 8 & 6 & 7 \\
\hline \multicolumn{7}{|l|}{ Business size: } \\
\hline - Average number of cows & 591 & 523 & 2,022 & 580 & 435 & 2,100 \\
\hline - Average number of heifers & 373 & 198 & 1,044 & 329 & 161 & 1,101 \\
\hline - Milk sold (million lbs) & 11.63 & 9.70 & 39.32 & 11.31 & 8.02 & 40.85 \\
\hline - FTE workers & 15 & 10 & 32 & 14 & 9 & 33 \\
\hline - Acres of pasture + cultivated land & 653 & 273 & 782 & 572 & 304 & 795 \\
\hline \multicolumn{7}{|l|}{ Production efficiency: } \\
\hline - Milk sold (Ibs / cow / year) & 17,549 & 18,283 & 19,133 & 17,603 & 17,734 & 19,646 \\
\hline - Cows / FTE worker & 37 & 55 & 61 & 39 & 47 & 67 \\
\hline - Milk sold / FTE worker (million Ibs) & 0.65 & 1.00 & 1.13 & 0.70 & 0.83 & 1.27 \\
\hline - Cull rate & $33 \%$ & $32 \%$ & $43 \%$ & $34 \%$ & $45 \%$ & $31 \%$ \\
\hline
\end{tabular}


Table 3.2. DBAP 2005 Summary - Revenues and expenses by net farm income per cwt and rate of return on assets (\$/cwt).

\begin{tabular}{|c|c|c|c|c|c|c|}
\hline \multirow[b]{3}{*}{ Category } & \multicolumn{3}{|c|}{ Net farm income (\$/cwt) } & \multicolumn{3}{|c|}{ Rate of return on assets (\%) } \\
\hline & \multicolumn{3}{|c|}{$\$(1.13)-$} & \multicolumn{3}{|c|}{$(3) \%-$} \\
\hline & $<\$(1.13)$ & $\$ 2.30$ & $>\$ 2.30$ & $<(3) \%$ & $9.5 \%$ & $>9.5 \%$ \\
\hline Number of farms & 7 & 7 & 7 & 8 & 6 & 7 \\
\hline \multicolumn{7}{|l|}{ Revenues: } \\
\hline Milk sold & 18.14 & 18.17 & 18.42 & 18.08 & 17.97 & 18.66 \\
\hline Raised, leased cow sales & 0.95 & 0.25 & 1.47 & 0.83 & 1.13 & 0.76 \\
\hline Heifer sales & 0.44 & 0.33 & 0.58 & 0.48 & 0.26 & 0.58 \\
\hline Gain on purchased livestock sales & $(0.48)$ & $(0.22)$ & 0.30 & $(0.62)$ & 0.46 & $(0.07)$ \\
\hline Other revenues & 2.27 & 0.84 & 0.72 & 2.03 & 1.00 & 0.66 \\
\hline Total revenues & 21.33 & 19.38 & 21.49 & 20.80 & 20.81 & 20.59 \\
\hline \multicolumn{7}{|l|}{ Expenses: } \\
\hline - Personnel & 4.60 & 3.11 & 2.80 & 4.48 & 3.13 & 2.70 \\
\hline - Purchased feed & 8.38 & 7.24 & 6.04 & 8.29 & 6.25 & 6.83 \\
\hline - Crops & 0.51 & 0.27 & 0.45 & 0.44 & 0.51 & 0.28 \\
\hline - Machinery & 1.56 & 0.84 & 0.94 & 1.43 & 0.95 & 0.88 \\
\hline - Livestock & 1.97 & 2.02 & 2.05 & 1.96 & 2.01 & 2.07 \\
\hline - Milk marketing & 1.27 & 1.11 & 1.30 & 1.19 & 1.26 & 1.23 \\
\hline - Buildings and land & 1.14 & 0.67 & 0.42 & 1.00 & 0.93 & 0.29 \\
\hline - Interest & 0.91 & 0.60 & 0.49 & 0.89 & 0.67 & 0.40 \\
\hline \multicolumn{7}{|l|}{ Depreciation: } \\
\hline - Livestock & 1.05 & 0.90 & 1.39 & 1.11 & 1.40 & 0.87 \\
\hline - Machinery & 1.15 & 0.78 & 0.52 & 1.03 & 1.04 & 0.37 \\
\hline - Buildings & 0.54 & 0.21 & 0.42 & 0.47 & 0.34 & 0.33 \\
\hline Other expenses & 1.22 & 1.04 & 0.77 & 1.22 & 0.87 & 0.89 \\
\hline Total expenses & 24.27 & 18.77 & 17.57 & 23.51 & 19.37 & 17.14 \\
\hline Net farm income from operations & $(2.94)$ & 0.60 & 3.92 & $(2.71)$ & 1.44 & 3.45 \\
\hline Gain on sale of capital assets & $(0.94)$ & 0.00 & $(0.46)$ & $(0.82)$ & $(0.53)$ & 0.00 \\
\hline Net farm income & $(3.87)$ & 0.61 & 3.47 & (3.53) & 0.91 & 3.45 \\
\hline
\end{tabular}


Table 3.3. DBAP 2005 Summary - Financial performance by net farm income per cwt and rate of return on assets.

\begin{tabular}{|c|c|c|c|c|c|c|}
\hline \multirow[b]{2}{*}{ Category } & \multicolumn{3}{|c|}{ Net farm income (\$/cwt) } & \multicolumn{3}{|c|}{ Rate of return on assets (\%) } \\
\hline & \multicolumn{3}{|c|}{$\$(1.13)-$} & $<(3) \%$ & (3)\% - & $>9.5 \%$ \\
\hline Number of farms & 7 & 7 & 7 & 8 & 6 & 7 \\
\hline \multicolumn{7}{|l|}{ Liquidity: } \\
\hline - Current ratio & 1.02 & 12.16 & 4.17 & 0.99 & 13.96 & 4.25 \\
\hline - Working capital $(\$)$ & $(307,705)$ & 24,654 & 652,257 & $(275,332)$ & 74,306 & 620,181 \\
\hline \multicolumn{7}{|l|}{ Solvency: } \\
\hline - Debt to asset ratio & 0.39 & 0.45 & 0.34 & 0.41 & 0.44 & 0.33 \\
\hline - Equity to asset ratio & 0.61 & 0.55 & 0.66 & 0.59 & 0.56 & 0.67 \\
\hline - Debt to equity ratio ${ }^{1}$ & 2.90 & 3.94 & 0.57 & 2.70 & 4.38 & 0.57 \\
\hline \multicolumn{7}{|l|}{ Profitability: } \\
\hline - Rate of return on assets & $(0.06)$ & 0.03 & 0.16 & $(0.06)$ & 0.03 & 0.17 \\
\hline - Rate of return on equity & $(0.27)$ & 0.10 & 0.24 & $(0.25)$ & 0.13 & 0.25 \\
\hline - Operating profit margin ratio & $(0.17)$ & 0.03 & 0.18 & $(0.15)$ & 0.05 & 0.18 \\
\hline \multicolumn{7}{|l|}{ Financial efficiency: } \\
\hline - Asset turnover rate & 0.55 & 0.90 & 0.89 & 0.57 & 0.82 & 0.98 \\
\hline - Operating expense ratio & 0.97 & 0.84 & 0.69 & 0.96 & 0.78 & 0.74 \\
\hline - Depreciation expense ratio & 0.13 & 0.10 & 0.10 & 0.13 & 0.13 & 0.08 \\
\hline - Interest expense ratio & 0.04 & 0.03 & 0.02 & 0.04 & 0.03 & 0.02 \\
\hline - NFIFO ratio ${ }^{2}$ & $(0.14)$ & 0.03 & 0.18 & $(0.13)$ & 0.06 & 0.17 \\
\hline \multicolumn{7}{|l|}{ Repayment capacity: } \\
\hline - Cash flow coverage ratio & 0.08 & 13.12 & 4.67 & 0.23 & 14.84 & 4.89 \\
\hline - Term debt coverage ratio ${ }^{3}$ & 1.26 & 0.82 & 6.61 & 1.21 & 0.48 & 6.90 \\
\hline - Capital replacement margin ${ }^{4}(\$)$ & $(324,083)$ & 60,030 & $1,228,600$ & $(291,751)$ & 74,639 & $1,234,001$ \\
\hline
\end{tabular}

${ }^{1}$ One dairy farm had negative equity.

${ }^{2}$ Net farm income from operations ratio.

${ }^{3}$ Term debt and capital lease coverage ratio.

${ }^{4}$ Capital replacement and term debt repayment margin. 
Table 3.4. DBAP 2005 Summary - Balance sheet by net farm income per cwt and rate of return on assets (\$/cow).

\begin{tabular}{|c|c|c|c|c|c|c|}
\hline \multirow[b]{3}{*}{ Category } & \multicolumn{3}{|c|}{ Net farm income (\$/cwt) } & \multicolumn{3}{|c|}{ Rate of return on assets (\%) } \\
\hline & \multicolumn{3}{|c|}{$\$(1.13)-$} & \multicolumn{3}{|c|}{ (3)\% - } \\
\hline & $<\$(1.13)$ & 2.30 & $>\$ 2.30$ & $<(3) \%$ & $9.5 \%$ & $>9.5 \%$ \\
\hline Number of farms & 7 & 7 & 7 & 8 & 6 & 7 \\
\hline \multicolumn{7}{|c|}{ Balance sheet (January 1): } \\
\hline - Current assets & 337 & 302 & 646 & 330 & 428 & 541 \\
\hline - Total assets & 10,601 & 4,794 & 5,004 & 9,766 & 6,119 & 3,993 \\
\hline - Current liabilities & 570 & 506 & 446 & 535 & 543 & 446 \\
\hline - Total liabilities & 1,930 & 1,828 & 1,671 & 1,950 & 1,982 & 1,502 \\
\hline - Equity & 8,672 & 2,966 & 3,332 & 7,816 & 4,137 & 2,491 \\
\hline \multicolumn{7}{|c|}{ Balance sheet (December 31): } \\
\hline - Current assets & 288 & 340 & 831 & 288 & 486 & 714 \\
\hline - Total assets & 9,884 & 4,634 & 5,486 & 9,153 & 5,952 & 4,441 \\
\hline - Current liabilities & 468 & 294 & 397 & 457 & 222 & 446 \\
\hline - Total liabilities & 2,078 & 1,791 & 1,819 & 2,106 & 2,017 & 1,553 \\
\hline - Equity & 7,806 & 2,843 & 3,666 & 7,048 & 3,935 & 2,888 \\
\hline
\end{tabular}


Table 4.1. DBAP 2005 Summary - Business size and production efficiency by assets per cow and liabilities per cow.

\begin{tabular}{|c|c|c|c|c|c|c|}
\hline \multirow[b]{2}{*}{ Category } & \multicolumn{3}{|c|}{ Assets (\$/cow) } & \multicolumn{3}{|c|}{ Liabilities (\$/cow) } \\
\hline & \multicolumn{2}{|r|}{$\$ 4,000-$} & $>\$ 5,600$ & $<\$ 1,340$ & $\$ 1,340$ & $>\$ 2,500$ \\
\hline Number of farms & 7 & 7 & 7 & 7 & 7 & 7 \\
\hline \multicolumn{7}{|l|}{ Business Size: } \\
\hline - Average number of cows & 1,167 & 1,331 & 638 & 1,212 & 1,049 & 874 \\
\hline - Average number of heifers & 710 & 471 & 434 & 886 & 142 & 588 \\
\hline • Milk sold (million lbs) & 22.87 & 24.49 & 13.28 & 24.24 & 17.69 & 18.71 \\
\hline - FTE workers & 22 & 18 & 17 & 27 & 14 & 17 \\
\hline - Acres of pasture + cultivated land & 426 & 558 & 725 & 608 & 424 & 677 \\
\hline \multicolumn{7}{|l|}{ Production Efficiency: } \\
\hline - Milk sold (lbs/cow/year) & 17,947 & 18,535 & 18,483 & 19,077 & 17,549 & 18,339 \\
\hline - Cows / FTE worker & 52 & 66 & 34 & 45 & 65 & 43 \\
\hline - Milk sold / FTE worker (million lbs) & 0.93 & 1.20 & 0.65 & 0.85 & 1.10 & 0.82 \\
\hline - Cull rate & $30 \%$ & $33 \%$ & $45 \%$ & $35 \%$ & $29 \%$ & $43 \%$ \\
\hline
\end{tabular}


Table 4.2. DBAP 2005 Summary - Revenues and expenses by assets per cow and liabilities per cow ( $\$ / c w t)$.

\begin{tabular}{|c|c|c|c|c|c|c|}
\hline \multirow[b]{3}{*}{ Category } & \multicolumn{3}{|c|}{ Assets ( $\$ /$ cow $)$} & \multicolumn{3}{|c|}{ Liabilities ( $\$ /$ cow) } \\
\hline & \multicolumn{3}{|c|}{$\$ 4,000$} & \multicolumn{3}{|c|}{$\$ 1,340$} \\
\hline & $<\$ 4,000$ & $-\$ 5,600$ & $>\$ 5,600$ & $<\$ 1,340$ & $-\$ 2,500$ & $>\$ 2,500$ \\
\hline Number of farms & 7 & 7 & 7 & 7 & 7 & 7 \\
\hline \multicolumn{7}{|l|}{ Revenues: } \\
\hline - Milk sold & 18.53 & 18.31 & 17.88 & 18.26 & 18.55 & 17.92 \\
\hline - Raised, leased cow sales & 0.51 & 0.78 & 1.39 & 0.79 & 0.05 & 1.84 \\
\hline - Heifer sales & 0.35 & 0.55 & 0.45 & 0.49 & 0.58 & 0.29 \\
\hline - Gain on purchased livestock sales & $(0.01)$ & $(0.58)$ & 0.20 & 0.04 & $(0.40)$ & $(0.04)$ \\
\hline - Other revenues & 0.80 & 1.30 & 1.73 & 1.03 & 1.02 & 1.78 \\
\hline - Total revenues & 20.18 & 20.37 & 21.65 & 20.61 & 19.80 & 21.79 \\
\hline \multicolumn{7}{|l|}{ Expenses: } \\
\hline - Personnel & 2.86 & 3.14 & 4.51 & 4.31 & 2.85 & 3.35 \\
\hline - Purchased feed & 7.64 & 7.88 & 6.14 & 6.95 & 6.90 & 7.81 \\
\hline - Crops & 0.15 & 0.35 & 0.73 & 0.58 & 0.25 & 0.40 \\
\hline - Machinery & 0.88 & 0.99 & 1.46 & 1.38 & 0.78 & 1.17 \\
\hline - Livestock & 2.30 & 1.37 & 2.37 & 2.60 & 1.67 & 1.76 \\
\hline - Milk marketing & 1.23 & 1.21 & 1.24 & 1.09 & 1.28 & 1.30 \\
\hline - Buildings and land & 0.73 & 0.48 & 1.01 & 0.88 & 0.56 & 0.79 \\
\hline - Interest & 0.51 & 0.59 & 0.90 & 0.13 & 0.62 & 1.24 \\
\hline \multicolumn{7}{|l|}{ Depreciation } \\
\hline - Livestock & 1.03 & 1.36 & 0.94 & 0.20 & 1.36 & 1.77 \\
\hline - Machinery & 0.60 & 0.54 & 1.30 & 0.97 & 0.66 & 0.81 \\
\hline - Buildings & 0.21 & 0.33 & 0.62 & 0.31 & 0.25 & 0.61 \\
\hline Other expenses & 1.01 & 0.84 & 1.18 & 1.09 & 0.94 & 1.00 \\
\hline Total expenses & 19.14 & 19.08 & 22.39 & 20.48 & 18.13 & 22.00 \\
\hline Net farm income from operations & 1.04 & 1.29 & $(0.73)$ & 0.13 & 1.67 & $(0.21)$ \\
\hline Gain on sale of capital assets & $(0.01)$ & $(0.15)$ & $(1.22)$ & $(0.78)$ & 0.13 & $(0.74)$ \\
\hline Net farm income & 1.03 & 1.13 & $(1.96)$ & $(0.65)$ & 1.80 & $(0.94)$ \\
\hline
\end{tabular}


Table 4.3. DBAP 2005 Summary - Financial performance by assets per cow and liabilities per cow.

\begin{tabular}{|c|c|c|c|c|c|c|}
\hline \multirow[b]{3}{*}{ Category } & \multicolumn{3}{|c|}{ Assets ( $\$ /$ cow $)$} & \multicolumn{3}{|c|}{ Liabilities ( $\$ /$ cow) } \\
\hline & \multicolumn{3}{|c|}{$\$ 4,000$} & \multicolumn{3}{|c|}{$\$ 1,340$} \\
\hline & $<\$ 4,000$ & $-\$ 5,600$ & $>\$ 5,600$ & $<\$ 1,340$ & $-\$ 2,500$ & $>\$ 2,500$ \\
\hline Number of farms & 7 & 7 & 7 & 7 & 7 & 7 \\
\hline \multicolumn{7}{|l|}{ Liquidity: } \\
\hline - Current ratio & 1.82 & 2.58 & 12.94 & 2.27 & 1.37 & 13.70 \\
\hline - Working capital (\$) & 234,881 & 240,634 & $(106,309)$ & 224,631 & 172,138 & $(27,563)$ \\
\hline \multicolumn{7}{|l|}{ Solvency: } \\
\hline - Debt to asset ratio & 0.50 & 0.43 & 0.25 & 0.14 & 0.53 & 0.51 \\
\hline - Equity to asset ratio & 0.50 & 0.57 & 0.75 & 0.86 & 0.47 & 0.49 \\
\hline - Debt to equity ratio ${ }^{1}$ & 6.02 & 0.92 & 0.47 & 0.19 & 4.11 & 3.11 \\
\hline \multicolumn{7}{|l|}{ Profitability: } \\
\hline - Rate of return on assets & 0.06 & 0.06 & 0.00 & 0.04 & 0.08 & 0.01 \\
\hline - Rate of return on equity & 0.02 & 0.08 & $(0.03)$ & 0.05 & 0.18 & $(0.16)$ \\
\hline - Operating profit margin ratio & 0.05 & 0.07 & $(0.07)$ & $(0.02)$ & 0.09 & $(0.02)$ \\
\hline \multicolumn{7}{|l|}{ Financial efficiency: } \\
\hline - Asset turnover rate & 1.13 & 0.81 & 0.39 & 0.77 & 0.96 & 0.61 \\
\hline - Operating expense ratio & 0.83 & 0.80 & 0.87 & 0.91 & 0.77 & 0.81 \\
\hline - Depreciation expense ratio & 0.09 & 0.11 & 0.13 & 0.07 & 0.12 & 0.14 \\
\hline - Interest expense ratio & 0.03 & 0.03 & 0.04 & 0.01 & 0.03 & 0.06 \\
\hline - NFIFO ratio ${ }^{2}$ & 0.05 & 0.06 & $(0.04)$ & 0.01 & 0.08 & $(0.01)$ \\
\hline \multicolumn{7}{|l|}{ Repayment capacity: } \\
\hline - Cash flow coverage ratio & 1.10 & 3.89 & 12.88 & 0.92 & 1.11 & 15.83 \\
\hline - Term debt coverage ratio $^{3}$ & 1.57 & 6.04 & 1.08 & 1.80 & 6.08 & 0.81 \\
\hline - Capital replacement margin ${ }^{4}(\$)$ & 458,418 & 684,602 & $(178,473)$ & 499,638 & 349,460 & 115,449 \\
\hline
\end{tabular}

${ }^{1}$ One dairy farm had negative equity.

${ }^{2}$ Net farm income from operations ratio.

${ }^{3}$ Term debt and capital lease coverage ratio.

${ }^{4}$ Capital replacement and term debt repayment margin. 
Table 4.4. DBAP 2005 Summary - Balance sheet by assets per cow and liabilities per cow (\$/cow).

\begin{tabular}{|c|c|c|c|c|c|c|}
\hline \multirow[b]{3}{*}{ Category } & \multicolumn{3}{|c|}{ Assets $(\$ /$ cow $)$} & \multicolumn{3}{|c|}{ Liabilities (\$/cow) } \\
\hline & \multicolumn{3}{|c|}{$\$ 4,000$} & \multicolumn{3}{|c|}{$\$ 1,340$} \\
\hline & $<\$ 4,000$ & $-\$ 5,600$ & $>\$ 5,600$ & $<\$ 1,340$ & $-\$ 2,500$ & $>\$ 2,500$ \\
\hline Number of farms & 7 & 7 & 7 & 7 & 7 & 7 \\
\hline \multicolumn{7}{|c|}{ Balance sheet (January 1): } \\
\hline - Current assets & 264 & 392 & 629 & 377 & 407 & 501 \\
\hline - Total assets & 3,194 & 4,380 & 12,825 & 9,048 & 3,622 & 7,730 \\
\hline - Current liabilities & 539 & 629 & 354 & 350 & 505 & 667 \\
\hline - Total liabilities & 1,442 & 1,830 & 2,157 & 571 & 1,783 & 3,075 \\
\hline - Equity & 1,752 & 2,550 & 10,668 & 8,477 & 1,838 & 4,655 \\
\hline \multicolumn{7}{|c|}{ Balance sheet (December 31): } \\
\hline - Current assets & 349 & 544 & 566 & 338 & 643 & 478 \\
\hline - Total assets & 3,397 & 4,636 & 11,970 & 8,657 & 3,986 & 7,360 \\
\hline - Current liabilities & 275 & 541 & 342 & 249 & 509 & 400 \\
\hline - Total liabilities & 1,614 & 1,972 & 2,103 & 621 & 1,921 & 3,147 \\
\hline - Equity & 1,784 & 2,665 & 9,867 & 8,037 & 2,065 & 4,213 \\
\hline
\end{tabular}

